\title{
Virtual Reality als Instrument zur Gewinnung von Nutzerfeedback zu Technologieszenarien am Beispiel urbaner Mobilität
}

\author{
Patrick Ruess (D) Nathalie Wingartz
}

Eingegangen: 24. Oktober 2019 / Angenommen: 30. Januar 2020 / Online publiziert: 13. Februar 2020 (C) Der/die Autor(en) 2020

Zusammenfassung In diesem Artikel wird eine Methode zur Gewinnung von Nutzerfeedback beschrieben, die eine Virtual-Reality-Applikation (VR) als Informationsträger für die Vermittlung der Befragungsinhalte einsetzt. Die Methode wurde im Rahmen des Forschungsprojekts ,2049: Zeitreise Mobilität“ des Fraunhofer Instituts für Arbeitswirtschaft und Organisation (IAO) angewendet, um Nutzermeinungen und -emotionen im Hinblick auf Mobilitäts-Zukunftsszenarien und -technologien zu erheben. Der Beitrag legt das Vorgehen bei der Entwicklung, der Durchführung und der Auswertung der Methodik dar und diskutiert die zugrundeliegenden Ideen, die sich im vorgestellten Ansatz wiederfinden. Zudem zeigt der Abgleich mit einer Vergleichsgruppe, welche an einer identischen Befragung ohne Stimulus teilgenommen hat, wie sich das immersive Erlebnis auf die Teilnehmer und die Befragungsergebnisse auswirkt. Dieser Vergleich bestätigt, dass keine grundsätzliche Beeinflussung durch die VR-Experience auftritt, es in Teilen aber zu einer Verzerrung kommt. Die Analyse zeigt aber auch, dass einzelne Gestaltungsmerkmale des VR-Films einen wesentlichen Einfluss auf das Entscheidungsverhalten der Teilnehmer haben können. Die Nutzung von VR-Applikationen erweist sich allerdings gerade bei praxisbezogenen Fragestellungen als sehr wertvoll, da sie die Ansprache von Probanden erleichtert, Sichtbarkeit auf Veranstaltungen und Messen schafft sowie zu einem Austausch zwischen Probanden und Befragten anregt.

Schlüsselwörter Virtuelle Realität · Technologieakzeptanz · Nutzertest · Produktentwicklung

\footnotetext{
P. Ruess $(\bowtie) \cdot$ N. Wingartz

Fraunhofer-Institut für Arbeitswirtschaft und Organisation IAO, Nobelstraße 12, 70569 Stuttgart, Deutschland

E-Mail: Patrick.Ruess@iao.fraunhofer.de

N. Wingartz

E-Mail: Nathalie.Wingartz@iao.fraunhofer.de
} 


\title{
Virtual Reality as an Instrument for Obtaining User Feedback on Technology Scenarios Using the Example of Urban Mobility
}

\begin{abstract}
The article describes a method for obtaining user feedback that uses a virtual reality application as an information carrier for conveying the survey content. The method was used within the research project "2049: A Ride into the Future of Transportation" of the Fraunhofer Institute for Industrial Engineering (IAO) to collect user opinions and emotions regarding future transportation scenarios and technologies. The paper presents the procedure for the development, implementation and evaluation of the methodology and discusses the underlying ideas in the approach presented. In addition, the comparison with a comparison group that participated in an identical survey without stimulus shows how the immersive experience affects the participants and the survey results. This comparison confirms that there is no fundamental influence by the VR experience, but that there is a distortion in parts. However, the analysis also shows that individual design features of the VR film can have a significant influence on the decision-making behaviour of the participants. The use of VR applications, however, proves to be very valuable, especially for practice-related questions, as it facilitates the approach of test persons, creates visibility at events and trade fairs and encourages an exchange between test persons and respondents.
\end{abstract}

Keywords Virtual reality $\cdot$ Technology acceptance $\cdot$ User testing $\cdot$ New product development

\section{Einleitung}

Die urbane Mobilität steht vor einem Paradigmenwechsel. Insbesondere den Städten stehen im städtischen Individualverkehr, als auch im öffentlichen Nahverkehr große Veränderungen bevor. Das starke Bevölkerungswachstum in urbanen Räumen und der damit verbundene Flächendruck führen $\mathrm{zu}$ einem hohen Urbanisierungsgrad in Ballungsräumen auf der ganzen Welt. Dies macht eine Anpassung unseres derzeitigen Mobilitäts- und Verkehrssystems notwendig. Auch andere Treiber tragen zu tiefgreifenden Veränderungen bei. Die internationale Sensibilisierung für ein nachhaltigeres Wirtschafts- und Gesellschaftssystem stellt neue Anforderungen an Mobilität und Verkehr. Auch wandeln sich zunehmend die Nutzungsbedürfnisse und fordern eine größere Flexibilität und Verlässlichkeit von Angeboten abseits des Individualverkehrs, die den ÖPNV komplementieren oder in Konkurrenz zu ihm treten können. Insbesondere privatwirtschaftliche Akteure wie Uber oder Lyft haben hierbei mit ihren Dienstleistungen eine Erwartungshaltung geweckt, wie nutzerzentrierte Mobilität aussehen könnte. Gleichzeitig stehen den Treibern der Veränderung neue Technologien und Angebote gegenüber, die diese neuen Anforderungen adressieren und potentielle Lösungen bieten. Hierzu zählen emissionsärmere Antriebsformen, alternative Betreiber- oder Finanzierungsmodelle, autonome Fahrzeuge sowie neue Sharing-Angebote, die beispielsweise E-Tretroller oder Leihräder im öffentlichen Raum bereitstellen. 
Ähnlich komplexe Veränderungen und Trends wie im Mobilitätssektor lassen sich für zahlreiche andere Handlungsfelder aufzeigen, sei es im Energiesektor, im Bereich der Pflege, oder im Bereich Arbeit und Industrie 40. All diesen Themenfeldern ist gemeinsam, dass die Zukunft offen ist und sich Fragen aufdrängen wie: werden wir zum Anhängsel von Computern und Robotern? Welche Tätigkeiten haben Bestand? Welche Technologien werden wir zukünftig nutzen?

Aus einer forschunsgpraktischen Sicht stellt sich in diesen breiten Handlungsfeldern häufig die Frage, wie sich erkennen lässt, wie und ob sich neue Technologien auch langfristig etablieren lassen. Wie lässt sich die Nutzerakzeptanz neuer Technologien, Konzepte und Ideen überprüfen, die bislang noch nicht existieren oder der großen Mehrheit der Nutzenden nicht bekannt ist? Gerade Ansätze mit hohem Innovationsgrad und längerem Entwicklungshorizont sind erklärungsbedürftig, was Produkttests oder Akzeptanzbewertungen zu einem komplexen Unterfangen machen. Die Entwicklung haptischer Prototypen als präferierte Herangehensweise ist kostspielig und bietet nur selten eine Funktionalität, die der späteren Leistungsfähigkeit des Produkts gerecht wird. Konzept-Beschreibungen und visuelle Methoden wie Grafiken oder Animationen erweisen sich als Grundlage für eine reflektierte Auseinandersetzung in einer Produktbewertung als abstrakt. Darüber hinaus sind sie in ihrer Aussagekraft limitiert, da begleitende Informationen in der Regel in Textform notwendig sind (Buckingham 2009; Lobinger und Mengis 2018).

Dieser Beitrag beschreibt daher ein Vorgehensmodell zur Erhebung von Nutzerfeedback, die auf einer durch Virtual Reality (VR) gestützten Applikation als wesentlichem Informationsträger basiert. Die Methodik wurde anhand des Anwendungsfalls von urbanen Mobilitäts-Zukunftskonzepten angewendet und wird im Rahmen dieses Artikels beschrieben und evaluiert. Ergänzend werden die Replizierbarkeit und Übertragbarkeit der Methodik auf Bereiche abseits der angewandten Forschung diskutiert.

Kap. 2 legt zunächst gängige Methoden der Nutzereinbindung bei der Produktund Technologieentwicklung in der Markt- und Konsumentenforschung sowie der Technologieakzeptanz-Forschung dar. Zudem wird anhand bestehender Veröffentlichungen aufgezeigt, welche Potentiale VR hierbei in der Interaktion mit Nutzern in einer frühen Phase der Produktentwicklung mit sich bringt und welche zugrundeliegenden Ansätze bei der Gestaltung entsprechender VR-Applikationen zum Tragen kommen. In Kap. 3 wird der Projektkontext, der Versuchsaufbau und die Vorgehensweise beschrieben.

Kap. 4 behandelt Erkenntnisse aus den Erhebungsdaten. Hierbei werden die Ergebnisse mit einer Teilnehmergruppe verglichen, die die identische Befragung ohne VR-Applikation durchgeführt haben. Somit können Aussagen über die Eignung der Methodik zur Gewinnung von Nutzerfeedback sowie zu den Vor- und Nachteilen in der Anwendung getroffen werden.

Im abschließenden fünften Kapitel wird ein Ausblick gegeben über die Möglichkeiten der zukünftigen Verwendung und Weiterentwicklung des Ansatzes sowie über die Anknüpfungspunkte und Limitationen der Herangehensweise.

Die gewählte männliche Form bei Personenbezügen in diesem Artikel bezieht sich immer zugleich auf weibliche und männliche Personen. Auf eine konsequente Doppelbezeichnung wurde zugunsten einer besseren Lesbarkeit verzichtet. 


\section{Möglichkeiten der Nutzereinbindung bei der Produkt- und Konzeptentwicklung}

Unternehmen verlassen sich bei der Entwicklung neuer Produkte und Dienstleistungen schon seit geraumer Zeit nicht mehr ausschließlich auf die eigenen Ressourcen und Ideen. Stattdessen haben sich in den vergangenen Jahren zahlreiche Ansätze und Vorgehensweisen etabliert, die sich zwar in Ursprung und Perspektive inhaltlich voneinander unterscheiden, die jedoch mit ihrer konsequenten, frühzeitigen und kontinuierlichen Beteiligung von Nutzern und Kunden eine wesentliche Gemeinsamkeit besitzen. Dieser institutionalisierten Einbindung und Nutzung externen Wissens für neue Innovationen und Produkte liegt das Open-Innovation-Konzept zu Grunde (Chesbrough 2010). Dieses zeigt sich auch in einer nutzer- und dienstleistungsorientierten Sichtweise, die sich in Forschung und Praxis unter dem Begriff Co-Creation durchgesetzt hat (Prahalad und Ramaswamy 2004). Co-Creation beschreibt eine gemeinschaftliche Wertschöpfung von Anbietern, Nutzern und weiteren Akteuren und lässt sich auch auf die Produkt- und Softwareentwicklung übertragen. Auch hier gelten Nutzerfeedback und Nutzerinteraktion in frühen, aber auch in fortgeschrittenen Phasen des Entwicklungsprozesses als wichtige Erfolgsfaktoren.

\subsection{Instrumente zur Nutzereinbindung und Akzeptanzbewertung bei der Produkt- und Konzeptentwicklung}

Es existieren unterschiedliche methodische Mittel bei der Einbindung und Gewinnung von Nutzerwissen im Produktentwicklungsprozess. Diese umfassen Interviewtechniken, Beobachtungen, Tests sowie die Beteiligung von Lead Usern (van Kleef 2006). Die Instrumente verfügen über eine unterschiedliche Eignung in den verschiedenen Phasen und Entwicklungsstadien neuer Produkte. In diesem Beitrag wird die vorgestellte Methodik anhand von Zukunftskonzepten beschrieben. Dies kommt einer Nutzereinbindung in einer sehr frühen Phase gleich, die beispielsweise als Concept Development oder Opportunity Identification (Ernst et al. 2010) beschrieben wird.

Darüber hinaus wurden auch zahlreiche Modelle und Ansätze entwickelt, die sich mit der Akzeptanz und Adoption neuer Technologien und Produkte befassen. Diese haben ihren Ursprung in der Soziologie sowie der Sozialpsychologie und wurden zu Beginn insbesondere im Umfeld betrieblicher IT-Systeme und -Technologien eingesetzt. Inzwischen finden sie jedoch auch in vielen weiteren Bereichen eine breite Anwendung (Taherdoost 2018). Zu den etabliertesten Ansätzen zählt das Technology Acceptance Model (TAM) nach Davis (1989), welches auch im Kontext von Mobilitäts-Innovationen und Technologien zur Personenbeförderung breite Verwendung erfährt. Die Akzeptanz gemäß TAM hängt von den Variablen der wahrgenommenen Nützlichkeit (Perceived Usefulness) und der wahrgenommenen Benutzerfreundlichkeit (Perceived Ease of Use) ab.

Im Rahmen der in diesem Beitrag vorliegenden Erhebung werden jedoch entferntere Mobilitäts-Konzepte und Zukunftsszenarien adressiert, die mit den sehr konkreten Variablen des TAM nicht bewertet werden können. Daher bezieht sich die Befragung auf die grundlegende Einstellung bzw. Affinität, die sich in den Mei- 
nungen und Emotionen der Nutzer zeigt. Die Einstellung eines Nutzers, die auch dem TAM zu Grunde liegt, wird als Indikator dafür definiert, wie sehr einer Person etwas gefällt oder wie sehr sie Gefallen findet am eigenen Verhalten in Bezug auf eine Sache (Edison und Geissler 2003).

\subsection{Potentiale durch VR in der Nutzereinbindung}

Die Visualisierung von Mock-ups oder Prototypen von noch nicht existierenden Produktentwürfen gehört zu den wichtigsten Anwendungsfällen virtueller Erlebnisräume und Umgebungen. Die Forschung fokussiert sich hierbei im Besonderen auf die Untersuchung des Konsumentenverhaltens mit Blick auf physische Reaktionen des menschlichen Körpers, die Rückschlüsse auf die Aufmerksamkeitsspanne, gewecktes Interesse, herbeigeführte Erschöpfung oder erzeugte Emotionen beim Probanden ermöglichen (Alcañiz et al. 2019). Nach dem Ansatz der Information Acceleration werden einem Nutzer in einer virtuellen Testumgebung alle Informationen zu einem zukünftigen Produkt oder Innovationskonzept zugänglich gemacht (Urban et al. 1996). Innerhalb dieses virtuellen Showrooms werden Interaktionsmöglichkeiten geschaffen, um Feedback zu erhalten. Die Methode findet in unterschiedlichen Branchen Anwendung und zielt auf die Generierung von Daten ab, mit denen sich Vermarktungspotentiale prognostizieren lassen (van Kleef 2006).

Neben dem rein visuellen Effekt können auch Narrative genutzt werden, um Wissen verfügbar zu machen. Gerade bei der Aufbereitung und Vermittlung wissenschaftlicher Inhalte für anwendungsbezogene Fragestellungen oder dem Transfer von Forschungsinhalten für ein nichtwissenschaftliches Zielpublikum bieten sich diesbezüglich Anknüpfungspunkte. Die Bereitstellung von Wissen in Form von Erzählungen oder einer konsistenten Handlung schafft eine Zugänglichkeit für Interessierte, die über konventionelle Kanäle nicht erreicht werden können (Dahlstrom 2014). VR eignet sich als Medium, um Narrative abzubilden.

Geschichten und Erzählungen in Zusammenhang mit einer Befragung können auch als Gamification-Element angesehen werden. Gamification bezeichnet die Verwendung spielerischer Elemente zur Motivation und Einbindung von Nutzern bei der Lösung von Problemen (Zichermann und Cunningham 2011). Die Gestaltung von „gamified surveys“ wirkt sich positiv auf Erhebungsergebnisse aus, was sich durch eine höhere Quote an vollständigen Antworten, geringeren Abbrecherzahlen oder übersprungenen Elementen bemerkbar macht. Die spielerischen Elemente stärken die Bindung des Nutzers, wecken Interesse und erzeugen Unterhaltung (Keusch und Zhang 2017).

\section{VR-gestützte Nutzerbefragung}

Als Untersuchungsgegenstand in diesem Beitrag dient eine breit angelegte Nutzerbefragung im Rahmen des Projekts ,2049: Zeitreise Mobilität“ des Fraunhofer Instituts für Arbeitswirtschaft und Organisation (IAO) und dem Hessischen Rundfunk. Ziel des Projekts war die Akzeptanzuntersuchung von Zukunftsszenarien und -technologien im Feld der urbanen Mobilität. Für die Erhebung wurde eine Virtual Reality- 
Applikation entwickelt, die den Teilnehmern die relevanten Inhalte im Vorfeld der Befragung visuell vermittelt.

\subsection{Methodische Vorgehensweise bei der Entwicklung und Durchführung}

Der Nutzer wird per Virtual Reality in ein Szenario realer Städte im Jahr 2049 geführt und lernt dort unterschiedliche Mobilitäts-Zukunftskonzepte kennen. Als wissenschaftlicher Partner des Projekts war das Fraunhofer IAO an der inhaltlichen Entwicklung der Szenarien beteiligt. Diese wurden im Anschluss in plausible Anwendungsszenarien überführt und dem Betrachter in einer konsistenten Geschichte erlebbar gemacht. Ergänzend zur Nutzererhebung führte das Fraunhofer IAO qualitative Interviews mit Mobilitäts-Experten durch und entwickelte hieraus eine Studie, welche Zukunftsbilder für das Mobilitäts- und Verkehrssystem von morgen zeichnet.

Die Nutzerbefragung fand im Zeitraum Mai bis Oktober 2019 statt und wurde auf mehreren Veranstaltungen in Deutschland und den USA durchgeführt. Mit der Erhebung konnte ein sehr breites Zielpublikum erreicht werden. Während auf der re:publica und der Internationalen Automobil-Ausstellung IAA (siehe Abb. 1) ein technisch versiertes und digital affines Zielpublikum angesprochen werden konnte, erreichten beispielsweise Präsenzen auf dem Hessentag 2019 und der Bundesgartenschau auch ältere Probanden, die über weniger Branchen- und Fachkenntnisse verfügten.

Begleitend zur VR-Befragung bestand zudem auch die Möglichkeit an der Erhebung durch die Beantwortung eines Online-Fragebogens teilzunehmen. Unabhängig der Erhebungsart wurden in beiden Fällen die gleichen Fragen mit den gleichen Begleittexten und -grafiken abgefragt. Den Online-Teilnehmern blieb jedoch die Möglichkeit verwehrt, die Hintergrundinformationen durch immersives Erleben des VR-Films kennenzulernen. Sie erhielten abseits des Fragebogens keine weiteren Informationen. Die niederschwellig zugängliche Online-Befragung als etablierte Erhebungsform stellte somit ein Pendant zum experimentellen Setup der VR-unterstützten Version dar. Die Vorgehensweise und die beiden unterschiedlichen Zugänge zur Befragung ermöglichen einen Vergleich der beiden Testgruppen und bieten somit Anknüpfungspunkte für eine nähere Betrachtung, wie sich die VR-gestützte Erhebung von der konventionellen Methode ohne Stimulus abgrenzt (siehe Kap. 4).

Das VR-Erlebnis nahm die Rolle eines Transfer-Mediums zwischen Forschung und Praxis ein. Abstrakte Konzepte und Szenarien konnten in eine virtuelle Umgebung übersetzt werden, in der die Inhalte intuitiv wahrgenommen werden können. Die Probanden wurden somit mittels VR dazu befähigt, Aussagen zu ihren Einstellungen und Emotionen zu treffen, auch wenn ihnen die zuvor vermittelten Inhalte bislang nicht bekannt oder vertraut waren.

Mit dem VR-Film als Medium gelang zudem die Ansprache verschiedener Nutzergruppen mit unterschiedlichen demografischen Merkmalen. Die einzige Anpassung, die für die Erhebung notwendig war, bestand aus der Entwicklung einer deutsch- und einer englischsprachigen Version, da gleichermaßen Veranstaltungen in Deutschland, als auch in den USA adressiert wurden. Entlang der Konzeption, Entwicklung und Durchführung des Projekts wurden wesentliche Prinzipien und Gestaltungsmerkmale zu Grunde gelegt, die im Folgenden beschrieben werden. 


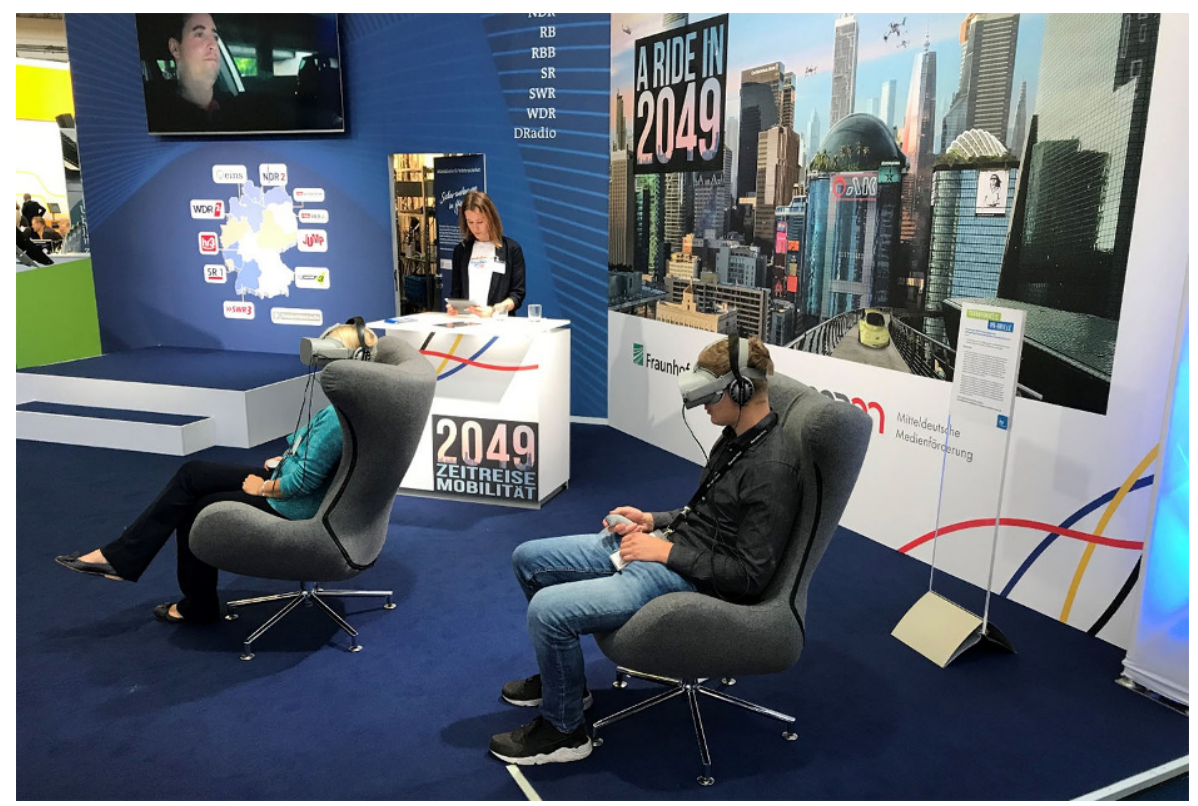

Abb. 1 Standaufbau der VR-Befragung auf der Internationalen Automobil-Ausstellung (IAA) 2019

\subsection{Auswahl von VR als Medium zur Gewinnung von Nutzerfeedback}

Die räumliche Mobilität des Menschen besitzt über alle gesellschaftlichen Ebenen hinweg eine große Relevanz, was bei entsprechenden Forschungsaktivitäten eine breit gestaltete Einbindung verschiedenster Nutzergruppen notwendig macht. Dies wird allerdings erheblich erschwert durch die unterschiedlichen Vorkenntnisse, die beispielsweise hinsichtlich des technologischen Verständnisses von neuen Mobilitätslösungen bestehen sowie bei sozioökonomischen und räumlichen Implikationen von Technologieinnovation. VR dient daher als Medium für die einfache Vermittlung von Begleitinformationen im Kontext erklärungsbedürftiger neuer Konzepte im Bereich Mobilität und bietet die Chance komplexe Inhalte leicht zugänglich zu machen. Unabhängig von Vorkenntnissen kann eine Visualisierung die Funktionalität und die Anwendungsmöglichkeiten neuer Ideen aufzeigen und somit als einheitliche Wissensgrundlage fungieren. Diese einheitliche Basis zu schaffen stellt gerade bei Zukunftskonzepten eine Herausforderung dar, da Räume für Interpretationen eröffnet werden. VR hingegen erschafft eine eindeutige und reproduzierbare Experimentierumgebung.

Zudem bieten VR-Applikationen auf Messen und Veranstaltungen Anreize, da sie durch den vermuteten Unterhaltungswert als attraktiv gelten. Somit bieten sie als ergänzender Stimulus im Vergleich zu konventionellen Methoden deutliche Vorteile bei der Ansprache potentieller Probanden. 
Tab. 1 Beschreibung der Szenarien

\begin{tabular}{|c|c|}
\hline Szenario & Beschreibung \\
\hline $\begin{array}{l}\text { (1) 3-D Mobili- } \\
\text { tät }\end{array}$ & $\begin{array}{l}\text { Der Wunsch nach individualisierter, bedarfsgerechter und effizienter Mobilität } \\
\text { bringt neue Transportkonzepte wie Passagierdrohnen und fliegende Autos hervor. } \\
\text { Durch diese 3D-Mobilität-Lösungen wird der Luftraum als weiterer Verkehrsträ- } \\
\text { ger für die innerstädtische Beförderung, aber auch für Reisen in die Umgebung, } \\
\text { erschlossen }\end{array}$ \\
\hline $\begin{array}{l}\text { (2) Autonomes } \\
\text { Fahren: }\end{array}$ & $\begin{array}{l}\text { In der Zukunft können sich Fahrzeuge autonom ohne menschlichen Eingriff fortbe- } \\
\text { wegen und sind daher in der Lage Fahrgäste selbstständig, effizient und sicher ans } \\
\text { Ziel zu bringen. Autonome Beförderungsmittel gehören zu einer innerstädtischen } \\
\text { Taxiflotte, die den Einwohnern bei Bedarf als Dienstleistung zur Verfügung stehen }\end{array}$ \\
\hline $\begin{array}{l}\text { (3) Kostenloser } \\
\text { OPPNV }\end{array}$ & $\begin{array}{l}\text { Für den öffentlichen Nahverkehr im Jahr } 2049 \text { wird es keine Fahrkarten mehr ge- } \\
\text { ben. Stattdessen wird die innerstädtische Beförderung kostenlos sein und durch } \\
\text { Werbeanzeigen und ergänzende Dienstleistungen finanziert, die den Fahrgästen } \\
\text { angeboten werden }\end{array}$ \\
\hline $\begin{array}{l}\text { (4) Urbane } \\
\text { Mikrokmobilität }\end{array}$ & $\begin{array}{l}\text { Kleinstfahrzeuge wie elektrische Roller oder Leihräder gehören in der Zukunft } \\
\text { zum Stadtbild jeder Metropole und sind das bevorzugte Verkehrsmittel für kurze } \\
\text { Wegstrecken. Die Fahrzeuge werden von den Einwohnern gemeinschaftlich genutzt }\end{array}$ \\
\hline $\begin{array}{l}\text { (5) Alternatives } \\
\text { Leben mit weni- } \\
\text { ger Mobilität }\end{array}$ & $\begin{array}{l}\text { Lebensräume werden in Zukunft so gestaltet sein, dass Menschen in ihrem un- } \\
\text { mittelbaren Umkreis alles vorfinden, was sie benötigen und sich wünschen. Das } \\
\text { individuelle Mobilitätsbedürfnis wird daher in der Zukunft viel geringer ausgeprägt } \\
\text { sein }\end{array}$ \\
\hline
\end{tabular}

\section{Storyboard, inhaltliche und grafische Umsetzung}

Mit dem Aufsetzen der VR-Brille beginnt für die Testpersonen eine Zeitreise, denn sie begeben sich direkt in deutsche und US-amerikanische Städte im Jahr 2049, die als virtuell begehbare Räume nachgebaut wurden. Begleitet werden Sie durch eine fiktive Figur, die die verschiedenen Szenen miteinander verknüpft, Orientierung bietet und das Gesehene inhaltlich einordnet. Durch die verzahnte Darstellung mehrerer Technologieanwendungsfälle in einem konsistenten Szenario können alternative oder in Konkurrenz zueinanderstehende Konzepte abgebildet werden. Die fünf Szenarien, die in „2049“ abgebildet und von den Teilnehmern durchlaufen wurden, werden in Tab. 1 beschrieben.

Der immersive Effekt virtueller Realität wurde genutzt, um die Wirkung von Technologien auf den umgebenden Raum zu visualisieren. Mit der Herstellung eines vertrauten Kontexts, beispielsweise durch bekannte räumliche Merkmale oder den Bezug zu derzeit bestehenden Mobilitätsangeboten, wurde ein Wiedererkennungswert geschaffen, durch den es den Probanden erleichtert wurde die Implikationen wahrzunehmen und zu bewerten. In ,2049“ wurde eine der größten Straßenkreuzungen Frankfurts aufgegriffen, die für das Szenario „Mikromobilität“ verkehrsberuhigt dargestellt und mit Sharing-Angeboten versehen wurde.

Informationen werden zum einen sprachlich durch die Begleitfigur vermittelt. Darüber hinaus werden wesentliche Charakteristiken zu einzelnen Zukunftsszenarien in Textform dynamisch angezeigt, wenn der Nutzer zugehörige Schlüsselobjekte im Blickfeld hat. Entlang des Handlungsfadens sind ausgewählte Interaktionsmöglichkeiten vorgesehen, die durch aktives Eingreifen für weitere Inhalte sensibilisieren. So kann zum Bespiel eine virtuelle Stadtkarte angepasst werden, um aufzuzeigen, 
Bitte bewerten Sie, wie realistisch Sie das Szenario finden.

\begin{tabular}{|cccccc|}
\hline $\begin{array}{c}1 \text { - sehr unrea- } \\
\text { listisch }\end{array}$ & 2 & 3 & 4 & $\begin{array}{c}5-\text { sehr realis- } \\
\text { tisch }\end{array}$ \\
Ihre Einschätzung & 0 & 0 & 0 & 0 & 0 \\
\hline
\end{tabular}

Bitte bewerten Sie, wie Ihnen persönlich das Szenario gefallen würde.

\begin{tabular}{|c|c|c|c|c|c|}
\hline & $\begin{array}{l}\text { 1- gefällt mir } \\
\text { gar nicht }\end{array}$ & 2 & 3 & 4 & $\begin{array}{l}5 \text { - gefällt mir } \\
\text { sehr }\end{array}$ \\
\hline Ihre Einschätzung & 0 & 0 & 0 & 0 & 0 \\
\hline
\end{tabular}

Abb. 2 Auszug aus dem Fragebogen zur Szenario-Bewertung

welche räumlichen Gestaltungsmöglichkeiten sich durch das autonome Fahren in urbanen Räumen zukünftig ergeben können. Der Ablauf der Handlung verändert sich durch den Nutzereingriff allerdings nicht.

\subsection{Befragung und Auswertung}

Die quantitative Befragung wurden mit den Probanden im Anschluss an das Erleben der VR-Experience mittels Tablets durchgeführt. Zum Einstieg wurde eine offene Frage gewählt, die losgelöst von den Szenarien auf die eigenen Wertvorstellungen und persönlichen Präferenzen beim Thema Mobilität abzielt: Wie sollte ihrer Meinung nach Mobilität im Jahr 2049 aussehen? Wählen Sie drei der unten aufgeführten Attribute, die Ihnen am wichtigsten sind!

Der Fragebogen wurde darauffolgend an der chronologischen Abfolge des Storyboards und den aufeinanderfolgenden Szenarien ausgerichtet. Um den Probanden weitere Orientierung zu geben und die Beantwortung der Fragen zu erleichtern, stellen die Szenarien aus Tab. 1 in sich geschlossene Fragegruppen dar, die immer nach dem gleichen Muster abgefragt wurden (siehe Abb. 2).

Im Anschluss an die Szenarienbeurteilung wurden Transferfragen gestellt, mit denen Einschätzungen zu möglichen Wechselwirkungen oder Abhängigkeiten zwischen verschiedenen Technologien oder Konzepten ermittelt werden können. Des Weiteren wurden persönliche Affinitäten und Einstellungen erfragt, die sich auf mehrere Szenarien gleichzeitig beziehen. Hierzu zählte die Bereitschaft zur Kontrollabgabe bei autonomen Fahrzeugen sowie die Offenheit für das Teilen von Fahrzeugen. Abschließend erfolgte die Erhebung von Paradaten zu demografischen Merkmalen.

\section{Ergebnisse}

Über den Befragungszeitraum hinweg haben insgesamt 1574 Teilnehmer den Fragenbogen abgeschlossen. 918 davon führten die Befragung im Anschluss an das VRErlebnis aus. 656 nahmen online teil. Dies entspricht einem Verhältnis von 58,3\% zu 41,7\% zu Gunsten von VR. Ein Vergleich dieser Verteilung hinsichtlich des Alters zeigt, dass mit VR insbesondere die jüngste Generation angesprochen wer- 
Tab. 2 Teilnehmer-Befragung absolut und relativ hinsichtlich VR und Online-Befragung

\begin{tabular}{llll}
\hline Teilnehmergruppe & VR & Online & VR-Anteil (\%) \\
\hline Teilnehmer insgesamt & 918 & 656 & 58,3 \\
Ab 1995 geboren (Generation Z) & 294 & 39 & 88,3 \\
1981-1994 (Generation Y) & 252 & 187 & 57,4 \\
1966-1980 (Generation X) & 201 & 183 & 52,3 \\
1956-1965 (Generation Babyboomer) & 111 & 156 & 41,6 \\
Bis 1955 (Generation Traditionals) & 46 & 90 & 33,8 \\
\hline
\end{tabular}

den konnte, während sich ältere Teilnehmer mehrheitlich online beteiligten (siehe Tab. 2).

Beim Geschlecht lässt sich hingegen keine starke Abweichung vom Gesamtverhältnis erkennen. 60,9\% der teilnehmenden Frauen und 56,8\% der Männer sahen die VR-Experience.

Zur Beantwortung des Fragebogens benötigten VR-Probanden mit 4:49 min deutlich weniger Zeit, als Teilnehmer ohne visuelle Stimulation $(6: 47 \mathrm{~min})$, bei einem Mittelwert über alle Befragungen von 5:36 min. Insgesamt steigt die Befragungszeit mit zunehmendem Alter an.

In Tab. 3 sind die Mittelwerte und die Standardabweichungen der Szenariobefragung abgebildet. Die Fragen wurden für jedes Szenario (siehe Tab. 1) nach dem in Abb. 2 gezeigten Fragen- und Skalenmuster erhoben.

Mit Blick auf die Mittelwerte lässt sich erkennen, dass Befragte mit VR-Experience keine grundsätzliche Tendenz aufweisen, wonach sie Szenarien positiver oder negativer einschätzen als Nicht-VR-Nutzer. Die Mittelwerte der einzelnen Fragen variieren zwischen den beiden Stichprobengruppen in den Szenarien (2) Autonomes Fahren, (3) Kostenloser ÖPNV und (4) Urbane Mikromobilität nur geringfügig. VR-Teilnehmende sehen allerdings das Szenario (5) Alternatives Leben mit weniger Mobilität gegenüber den Online-Teilnehmern als realistischer an. Bei der persönlichen Affinität für dieses Zukunftsbild ist ein solcher Unterschied zwischen beiden Gruppen wiederum nicht zu erkennen. Auffällig ist zudem insbesondere das Befragungsergebnis von Szenario (1) 3D-Mobilität, welches unter VR-Befragten auf deutlich positivere Resonanz stößt und auch als deutlich realistischer eingeschätzt wird.

Um zu überprüfen, ob sich die Bewertungen der Befragten bezüglich Realismus und Präferenz der Szenarien nach Befragungsgruppe (mit VR/ohne VR) signifikant unterscheiden, wurde zusätzlich ein Mann-Whitney-U-Test berechnet (siehe Tab. 4). Dieser Test ist geeignet für unverbundene und nicht normalverteilte Stichproben. Bei einem $P$-Wert von weniger als 0,05 ist ein signifikanter Unterschied gegeben. Für jene Fälle wurde zusätzlich die Effektstärke (Cohen's d) ermittelt, um abzuleiten, wie stark der Effekt auf die Verteilung der Antworten ausfällt, wenn Befragte zuvor die die VR-Experience gesehen hatten. Ein Effekt ist ab einem Wert von 0,2 (schwach) vorhanden. Ab 0,5 liegt ein mittlerer - und ab 0,8 ein starker Effekt vor.

Der Vergleich bestätigt, dass keine grundsätzliche Beeinflussung durch die VRExperience auftritt, es in Teilen aber zu einer leichten Verzerrung kommt. Mehrheitlich gibt es jedoch keine wesentlichen Unterschiede und Effekte zwischen den 


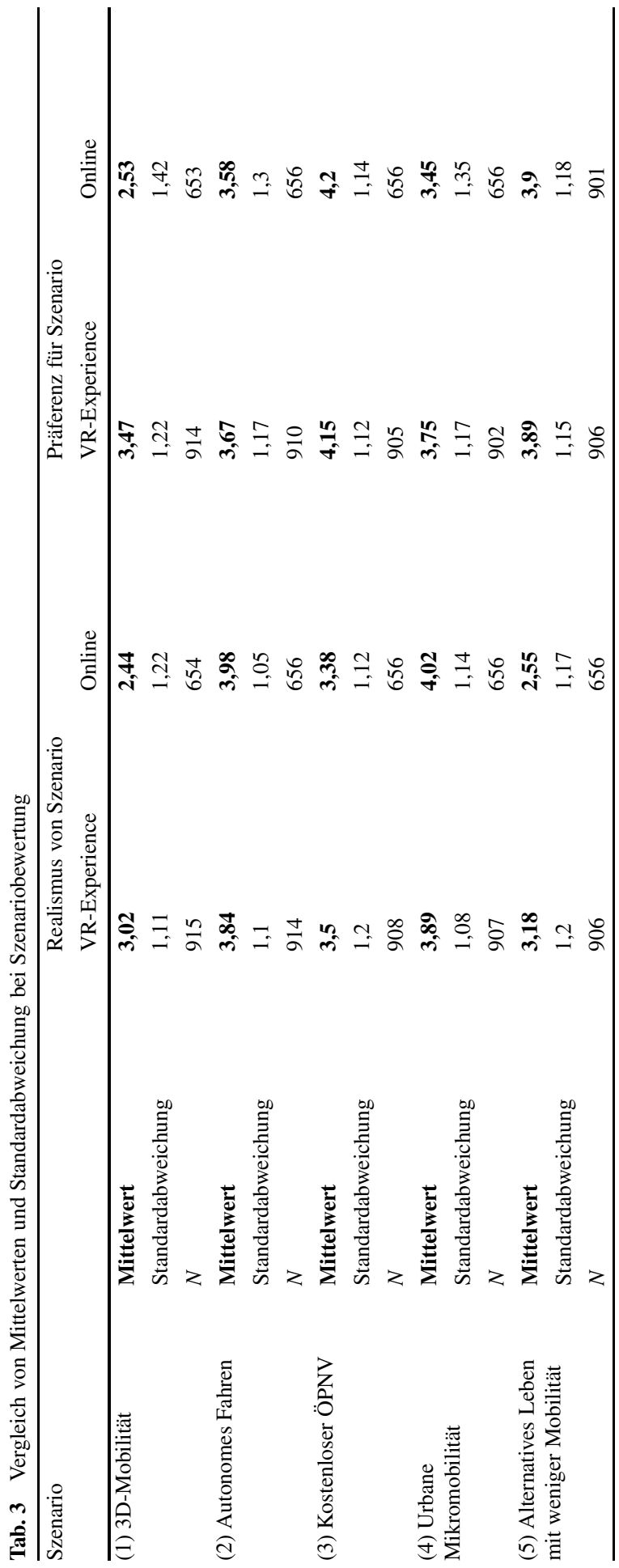


Tab. 4 Vergleich von Stichprobengruppen

\begin{tabular}{|c|c|c|c|}
\hline \multicolumn{2}{|l|}{ Szenario } & \multirow{2}{*}{$\begin{array}{l}\text { Realismus von Szenario } \\
\mathbf{0 , 0 0}\end{array}$} & \multirow{2}{*}{$\begin{array}{l}\text { Präferenz für Szenario } \\
\mathbf{0 , 0 0}\end{array}$} \\
\hline (1) 3D-Mobilität & $\begin{array}{l}\text { Mann-Whitney-U } \\
\text { ( } p \text {-Wert) }\end{array}$ & & \\
\hline & Cohen's d & 0,5 (medium) & 0,7 (medium) \\
\hline \multirow[t]{2}{*}{ (2) Autonomes Fahren } & $\begin{array}{l}\text { Mann-Whitney-U } \\
\text { ( } p \text {-Wert) }\end{array}$ & 0,01 & 0,41 \\
\hline & Cohen's d & $-0,1$ (negligible) & - \\
\hline \multirow[t]{2}{*}{ (3) Kostenloser ÖPNV } & $\begin{array}{l}\text { Mann-Whitney-U } \\
\text { ( } p \text {-Wert) }\end{array}$ & $\mathbf{0 , 0 5}$ & 0,17 \\
\hline & Cohen's d & 0,1 (negligible) & - \\
\hline \multirow[t]{2}{*}{$\begin{array}{l}\text { (4) Urbane } \\
\text { Mikromobilität }\end{array}$} & $\begin{array}{l}\text { Mann-Whitney-U } \\
\text { ( } p \text {-Wert) }\end{array}$ & $\mathbf{0 , 0 0}$ & $\mathbf{0 , 0 0}$ \\
\hline & Cohen's d & $-0,1$ (negligible) & 0,2 (small) \\
\hline \multirow[t]{2}{*}{$\begin{array}{l}\text { (5) Alternatives Leben } \\
\text { mit weniger Mobilität }\end{array}$} & $\begin{array}{l}\text { Mann-Whitney-U } \\
\text { ( } p \text {-Wert) }\end{array}$ & $\mathbf{0 , 0 0}$ & 0,64 \\
\hline & Cohen's d & 0,5 (medium) & - \\
\hline
\end{tabular}

beiden Testgruppen, so dass eine kombinierte Auswertung beispielsweise in den Szenarien (2) und (3) möglich ist.

Die Analyse macht aber auch deutlich, dass einzelne Gestaltungsmerkmale des VR-Films einen Einfluss auf das Entscheidungsverhalten der Teilnehmer haben können, wie anhand der sichtbaren Differenzen in den Mittelwerten (siehe Tab. 3) und der Effektstärken (siehe Tab. 4) im Fall von Szenario (1) 3D-Mobilität und bei der Einschätzung des Realismus beim Thema (5) Alternatives Leben zu erkennen ist. Dies lässt sich durch eine neutrale Konzeption der VR-Erlebnissen in Verbindung mit Vorabtests durch verschiedene Kontrollgruppen ausräumen.

\section{Diskussion}

VR-gestützte Befragungen sind ein bislang unterschätztes Instrument bei der Einbindung von Nutzern und bei der Gewinnung von Feedback für zukünftige Konzepte, Produkte und Ideen. In diesem Beitrag werden aus anwendungsorientierter Sicht die Vorgehensweise und die Erkenntnisse aus der Konzeption, der Anwendung und den Ergebnissen einer solchen Erhebung dargelegt. Der Artikel leistet hiermit zwei Beiträge zum Thema „Forschung für die Praxis“. Zum einen greift die Beschreibung einer Methodik zur Vermittlung von wissenschaftlichen Inhalten für eine nicht-wissenschaftliche Zielgruppe den Wissenstransfer zwischen Forschung und Praxis auf. Darüber hinaus lassen sich VR-gestützte Erhebungen als Methodik auch abseits der Forschung einsetzen und besitzen daher Relevanz für Praktiker beispielsweise in der Produktentwicklung, dem Marketing oder dem Innovationsmanagement.

Die Nutzung von VR-Applikationen, insbesondere bei praxisbezogenen Frageund Zielstellungen, erweist sich als sehr wertvoll, da sie die Ansprache von Probanden erleichtert, Sichtbarkeit auf Veranstaltungen und Messen schafft sowie zu einem Austausch zwischen Probanden und Befragten anregt. Das immersive Erleb- 
nis verstärkt eine aktive Auseinandersetzung der Teilnehmer mit den Inhalten und blendet ablenkende Störfaktoren, wie beispielsweise das eigene Smartphone in der unmittelbaren Umgebung, aus.

Ungeachtet dieser Potentiale, die die Methodik bietet, muss die Rolle des „Primings" durch die VR-Sequenz tiefergehend untersucht werden. Priming bezeichnet eine reizbedingte Beeinflussung, die implizite Gedächtnisinhalte aktiviert und meist unbewusste Assoziationen herstellt (Myers et al. 2014). Die Ergebnisse zeigen Verzerrungen, die durch VR hervorgerufen wurden, was zur Folge hat, dass die Forschungsergebnisse differenziert betrachtet werden müssen. Bei Anwendungsfällen mit größerem Praxisbezug besteht dahingehend aber sicherlich eine größere „Toleranzschwelle“, da hier oftmals auch schon ein Feedback in Form grundlegender Tendenzen oder die Identifikation von wesentlichen Trends nutzenstiftend ist. Weiterführend muss in zukünftigen Studien auch auch der Einfluss weiterer visueller Methoden wie konventionellen Videos als Stimuli näher betrachtet werden, um die Wirkung des immersiven Erlebnisses präziser definieren zu können. Dies konnte im Rahmen des durchgeführten Projekts nicht näher untersucht werden und stellt daher eine Limitation dar.

Funding Open Access funding provided by Projekt DEAL.

Open Access Dieser Artikel wird unter der Creative Commons Namensnennung 4.0 International Lizenz veröffentlicht, welche die Nutzung, Vervielfältigung, Bearbeitung, Verbreitung und Wiedergabe in jeglichem Medium und Format erlaubt, sofern Sie den/die ursprünglichen Autor(en) und die Quelle ordnungsgemäß nennen, einen Link zur Creative Commons Lizenz beifügen und angeben, ob Änderungen vorgenommen wurden.

Die in diesem Artikel enthaltenen Bilder und sonstiges Drittmaterial unterliegen ebenfalls der genannten Creative Commons Lizenz, sofern sich aus der Abbildungslegende nichts anderes ergibt. Sofern das betreffende Material nicht unter der genannten Creative Commons Lizenz steht und die betreffende Handlung nicht nach gesetzlichen Vorschriften erlaubt ist, ist für die oben aufgeführten Weiterverwendungen des Materials die Einwilligung des jeweiligen Rechteinhabers einzuholen.

Weitere Details zur Lizenz entnehmen Sie bitte der Lizenzinformation auf http://creativecommons.org/ licenses/by/4.0/deed.de.

\section{Literatur}

Alcañiz M, Bigné E, Guixeres J (2019) Virtual reality in marketing: a framework, review, and research agenda. Front Psychol 10:1530. https://doi.org/10.3389/fpsyg.2019.01530

Buckingham D (2009) "Creative" visual methods in media research: possibilities, problems and proposals. Media Cult Soc 31(4):633-652. https://doi.org/10.1177/0163443709335280

Chesbrough HW (2010) Open innovation. The new imperative for creating and profiting from technology. [Nachdr]. Harvard Business School Press, Boston

Dahlstrom MF (2014) Using narratives and storytelling to communicate science with nonexpert audiences. Proc Natl Acad Sci USA 111(Suppl 4):13614-13620. https://doi.org/10.1073/pnas.1320645111

Davis FD (1989) Perceived usefulness, perceived ease of use, and user acceptance of information technology. Mis Q 13(3):319. https://doi.org/10.2307/249008

Edison SW, Geissler GL (2003) Measuring attitudes towards general technology: antecedents, hypotheses and scale development. J Target Meas Analysis Mark 2003(12):137-156

Ernst H, Hoyer WD, Rübsaamen C (2010) Sales, marketing, and research-and-development cooperation across new product development stages: implications for success. J Mark 74(5):80-92. https://doi. org/10.1509/jmkg.74.5.80 
Keusch F, Zhang C (2017) A review of issues in gamified surveys. Soc Sci Comput Rev 35(2):147-166. https://doi.org/10.1177/0894439315608451

van Kleef PW (2006) Consumer research in the early stages of new product development. Issues and applications in the food domain

Lobinger K, Mengis J (2018) Visuelle Methoden. In: Lobinger K (Hrsg) Handbuch Visuelle Kommunikationsforschung, Bd. 28. Springer, Wiesbaden, S 1-24 (Springer Reference Sozialwissenschaften)

Myers DG, Hoppe-Graff S, Keller B (2014) Psychologie Bd. 3. Springer, Berlin https://doi.org/10.1007/ 978-3-642-40782-6

Prahalad CK, Ramaswamy Venkat (2004) Co-creation experiences: the next practice in value creation. J Interact Mark 18(3):5-14. https://doi.org/10.1002/dir.20015

Taherdoost H (2018) A review of technology acceptance and adoption models and theories. Procedia Manuf 22:960-967. https://doi.org/10.1016/j.promfg.2018.03.137

Urban GL, Weinberg BD, Hauser JR (1996) Premarket forecasting of really-new products. J Mark 60(1):47. https://doi.org/10.2307/1251887

Zichermann G, Cunningham C (2011) Gamification by design. Implementing game mechanics in web and mobile apps. 1. Aufl. O’Reilly, Sebastopol 